\title{
Thumbnail strain during pressing and plucking of a string
}

\author{
Satoshi SHIMAWAKI*, Atsushi NAKAYAMA*, Masataka NAKABAYASHI* and Naotaka SAKAI* \\ *Department of Mechanical and Intelligent Engineering, Utsunomiya University \\ 7-1-2 Yoto, Utsunomiya-shi, Tochigi 321-8585, Japan \\ E-mail: simawaki@cc.utsunomiya-u.ac.jp
}

Received 17 July 2014

\begin{abstract}
Human fingernails play an important role in daily life and while playing instruments. We previously showed that the strain generated at the radial and ulnar parts of the nail was asymmetric when the thumb pad was pressed down on a flat surface. The purpose of the present study was to investigate whether nail strain is asymmetrical while pressing or plucking a string with the thumb. Biaxial strain gauges were attached to the surface of the thumbnail of 15 subjects; the gauges were attached to the proximal-radial, distal-central, and proximal-ulnar parts of the thumbnail. We measured the maximum strain generated while pressing or plucking a string with the thumb pad. The strain values obtained were then compared among the measurement locations. We also measured the effects of contact angles and contact points on the thumbnail strain. Upon investigating the effect of the measurement locations, we found that during string-pressing motion, the maximum strain was significantly lower in the proximal-radial-axial direction than in the distal-central-axial and proximal-ulnar-axial directions. This indicated that an asymmetric strain generated at the proximal-radial and ulnar sides. We also investigated the effect of the contact angle and found that the maximum strain varied with changes in contact angle. The investigation of the effect of the contact point revealed that the maximum strain during string-pressing motion varied with changes in the contact point, although no change was observed during string plucking. Asymmetrical thumbnail strain may have been caused by the asymmetrical nature of the flexor tendon insertion and the distal phalangeal tuberosity of the thumb. Uniform pressure applied to the thumb pad became asymmetric in the radioulnar direction because of the interaction between the thumb pulp, distal phalanx, and nail. This asymmetry may then have been transmitted to the nail.
\end{abstract}

Key words : Nail, Strain, Thumb, Ungual pulp, Human engineering

\section{Introduction}

Human fingernails play an important role in daily life. When picking up small objects, the fingerpad deforms flexibly in order to grasp the object (Kapandji, 2007). The nail exists to efficiently transmit the pressure from the finger to the small object by suppressing the deformation of the fingerpad. This is particularly useful when doing tasks such as pressing the buttons on a cell phone (Sakai and Shimawaki, 2010). The nail also plays a role in picking up small objects, scratching object surfaces, and protecting the distal phalanx.

In addition to their importance in daily life, fingernails play an important role in playing musical instruments that require the use of the fingers, such as pushing securely down on flute holes with the fingerpads. Some acoustic guitar playing styles, such as arpeggio and finger picking, also require the use of the fingers and nails to pluck the strings. The four fingers from the thumb to the ring finger are usually used in such cases, with the thumb being responsible for the plucking of the $4^{\text {th }}, 5^{\text {th }}$, and $6^{\text {th }}$ strings. When playing in this manner, a large amount of pressure is thought to be applied to the nail. Playing musical instruments using fingernails is important in terms of operation, and it has not shown much change over centuries.

The nail is a semi-transparent, horny plate with a distal free edge, a proximal hidden edge, and two lateral edges (Ditre and Howe, 1992; Fleckman and Allan, 2001). The nail also has a slight curvature in the transverse and axial directions (Forslind, 1970; Murdan, 2011). The proximal portion of the nail is embedded under the skin, and a 
crescent-shaped lunula can be seen in this area as a result of underdeveloped keratinization. This inside of the nail is in close contact with the nail bed, which is in contact with the lateral tubercle and the lateral interosseous ligament of the distal phalanx; these hold the nail securely in place. A number of studies investigating the anatomical structure of the nail have shown that the nail is composed of a three-layer fibrous structure, with the thickest middle layer growing in the transverse direction (Lewin, 1965; Jarrett and Spearman, 1966; Baden, 1970; Farren et al., 2004). Jemec and Serup (1989) measured nail thickness with ultrasound and concluded that the nail is formed of a dried surface layer and a moist inner layer. Farren et al. (2004) investigated the dynamic properties of the nail by measuring the direction of fracture with a tear test and breaking strength with scissor cuts using human nail clippings. Farran et al. $(2008,2009)$ found that fractures occur easily in the transverse direction, which is the nail fiber direction, and that breaking strength in the transverse direction is the half of the breaking strength in the axial direction. They also demonstrated that the water content of the nail affects its physical properties.

Many studies on nail structure, such as those mentioned above, have been conducted; however, very few have investigated nail properties when the fingerpad is deformed. Using near-infrared light emitting diodes and photodetectors attached to the nail surface, Mascaro and Asada $(2001,2004)$ measured the color changes on the nail surface when the fingerpad was pushed down on objects. They found a correlation between the level of pressure applied to the fingerpad and the color of the nail. Nail color changes occur as a result of capillaries within the finger apex being compressed by pressure on the nail and finger apex, thereby disrupting blood flow and causing blood to pool in the area. In addition, using finite element analysis, Shimawaki and Sakai (2007) investigated fingertip deformation when the finger is pressed down on a flat surface; they discovered that the nail is compressed from both sides by the deformed fingerpad. By attaching biaxial strain gauges to three locations on the surface of the thumbnail, Shimawaki et al. (2006) also assessed the relationship between the amount of nail strain and the compression force exerted when the thumb is pressed down on a flat surface. A noteworthy finding of this study was the level of asymmetry of the thumbnail strain. The level of strain in the transverse direction in the proximal-radial part of the nail was shown to be significantly greater than that in the proximal-ulnar part.

In a previous report (Shimawaki et al., 2006), to develop a robotic hand or an electric artificial hand with nails that can respond to various movements (object control, machine operation, etc.), we investigated the mechanical characteristic of the thumb nail. From this study, we obtained the relationship between the amount of nail strain that occurs when pressing a flat plate and the pressing force. However, as mentioned earlier, the hand has various functions other than pressing a flat plate. The aim of the present study was to investigate whether nail strain became asymmetrical when a string is pressed or plucked with the thumb. This situation can be likened to the movement required when picking up a cylinder having a small diameter. In our previous study, we focused on the static or quasi-static changes in fingerpads. But, in this research, we also target the changes in the fingerpads while playing an instrument. As described in a previous report, biaxial strain gauges were attached to three locations on the thumbnail. The maximum strain achieved while pressing or plucking a string with the thumb pad was measured, and the values obtained at each measurement location were compared. On the basis of the results from both the previous and current studies, we estimate the functions of the thumb and thumb nail and consider the application of these results in the development of robotic and electric artificial hands.

\section{Methods}

\subsection{Subjects and experimental equipment}

Fifteen men confirmed to have no trauma or disease in the nails or the distal portion of the interphalangeal joint (hereinafter, IP joint) of the thumb of the right hand participated in this study. The subject's age ranged from 21 to 24 years. The mean and standard deviation values for other parameters were as follows: height $173 \pm 6.7 \mathrm{~cm}$, weight 66.0 $\pm 8.6 \mathrm{~kg}$, body fat percentage $16.6 \% \pm 4.7 \%$, and body mass index $21.8 \pm 2.5 \mathrm{~kg} / \mathrm{m}^{2}$. The length of the thumbnail was $14.5 \pm 1.4 \mathrm{~mm}$, the width was $14.6 \pm 1.4 \mathrm{~mm}$, and the thickness was $0.7 \pm 0.1 \mathrm{~mm}$. All of the subjects were right-handed, and none of them were guitar players. This study was approved by Ethical Review Committee on Research Involving Human Subject of Utsunomiya University prior to its commencement.

The sixth string (NS116 E-6 ${ }^{\text {th }}$ string; Yamaha Corp., Shizuoka, Japan) of a classical guitar was used for the experiment (Fig. 1). The string was made of nylon and had a diameter of $1.13 \mathrm{~mm}$. One end of the string was tied to the guitar bridge. The string was supported by the saddle and the nut, with the distance between them being $650 \mathrm{~mm}$. 


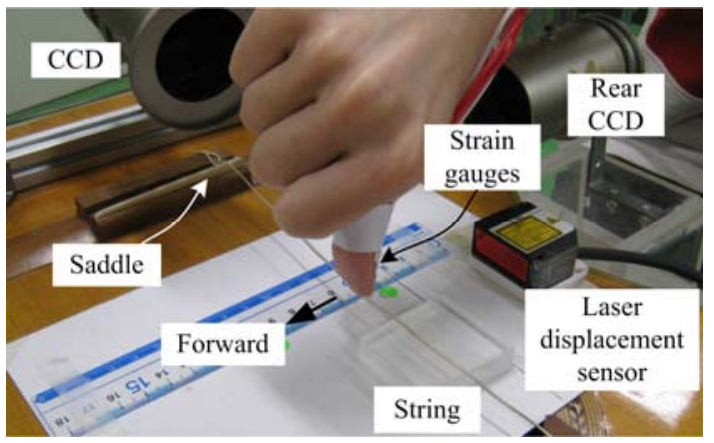

Fig.1 Experimental device.

Unlike a normal guitar string that is connected to a guitar peg, the string on the present experimental setup was instead dropped down vertically from the machine head to ensure that the initial string tension was constant in all experiments. A load of $78.4 \mathrm{~N}(8 \mathrm{~kg})$ was applied to this end of the string. This load generated the typical tension applied to the sixth string. The experimental setup was designed so that as the thumb pressed and plucked the string, its movement position was $120 \mathrm{~mm}$ from the saddle. We captured the thumb movement by 2 charge-coupled device (CCD) cameras placed at the rear and side of the thumb. A marker was attached to the string $10 \mathrm{~mm}$ away from the portion where the thumb movements would be performed. To calculate the amount of string movement, the amount of horizontal movement of the marker was measured using a laser displacement sensor (ZX-LD100; Omron Corp., Kyoto, Japan).

\subsection{Strain measurement location}

Three biaxial strain gauges (KFG-1-120-D16-23; Kyowa Electronic Instruments Ltd., Tokyo, Japan) were attached to the right thumbnail using a special cyanoacrylate adhesive (Fig. 2a). The grid size of the strain gauges was $1.1 \mathrm{~mm}$ in width and $1 \mathrm{~mm}$ in length. The diameter of the base was $5 \mathrm{~mm}$, and the thickness was $13 \mu \mathrm{m}$. Prior to attachment, the subjects were asked to clip off any unnecessary parts of the nail. The biaxial strain gauges used in the experiment could measure strain in two perpendicular directions simultaneously. One gauge each was attached proximal-radial, distal-central, and proximal-ulnar parts of the thumbnail. In the transverse direction, the nail was divided horizontally into three equal parts and gauges were placed in the center of each of these parts. Each gauge axis was placed to coincide with the axial and transverse directions of the nail. Consequently, in the present experiment, we measured the level of strain in six directions ( 2 directions each at 3 locations on the thumb nail surface). These directions were the proximal-radial-axial direction (1), the proximal-radial-transverse direction (2)), the distal-central-axial direction (3), the distal-central-transverse direction (4), the proximal-ulnar-axial direction (5), and the proximal-ulnar-transverse direction (6). Strain gauge output data were collected by a strain measurement interface (PCD-300A; Kyowa Electronic Instruments Ltd.) and recorded onto a personal computer.

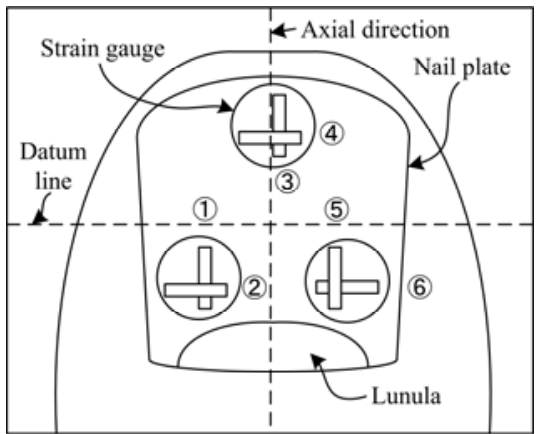

(a) Strain measurement location

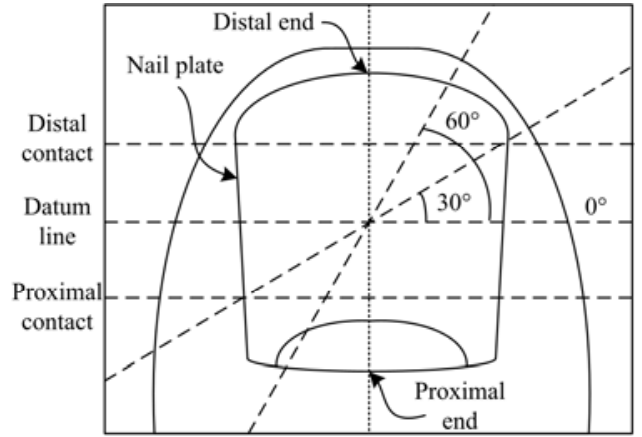

(b) String contact

Fig.2 (a) Three $0^{\circ} / 90^{\circ}$ biaxial strain gauges were affixed on the proximal-radial, distal-central, and proximal-ulnar parts of the thumb nail plate. (b) Five patterns for the string contact: contact angles of $0^{\circ}, 30^{\circ}$, and $60^{\circ}$ as well as distal and proximal contacts. 


\subsection{Method of contact with the string}

Subjects faced the string so that the string was parallel to their coronal plane. The subjects then pointed the tip of their right thumb downward, moved their thumb to the movement position, and adjusted the position so that the longitudinal axis of the thumb was vertical, using the images from the CCD cameras as reference. At this point, the IP joint was extended. The line perpendicular to the longitudinal axis of the thumb passing through the center of the nail plate was designated as the contact line. The thumb pad and the string were brought into contact by moving the thumb forward.

We defined two parameters for the contact between the string and thumb, i.e., contact angle and contact point. We designated the line that was perpendicular to the longitudinal axis of the thumb and passed through the center of the nail plate as the datum line. We defined the contact angle as the angle between the datum line and the string, which was modified by rotating the forearm. We conducted the present experiment at contact angles of $0^{\circ}, 30^{\circ}$, and $60^{\circ}$ and adjusted the contact points by moving the thumb parallel to the string distal or proximal to the datum line at a contact angle of $0^{\circ}$. The subjects readjusted the contact point using the images provided by the rear CCD camera as a reference. When the contact points were centered between the datum line and the distal or proximal ends of the nail plate, these points were designated as the distal and proximal contact points, respectively.

This arrangement gave us five patterns for the string contact (contact angle of $0^{\circ}, 30^{\circ}$ and $60^{\circ}$ and distal contact, and proximal contact, as shown in Fig. 2b). We defined the reference contact as the contact condition where the contact angle was $0^{\circ}$ and the datum line passed through the center of the nail plate.

\subsection{Experimental movements}

Two types of movement were performed in the present experiment. We first instructed each subject to place the thumb in the movement position without touching the string. For the string-pressing movement, subjects were instructed to press the string forward $10 \mathrm{~mm}$ for one second and then return the thumb to its original position for the next second, timed with a metronome. For the plucking movement, subjects were instructed to fix their IP joint and pluck the string by rotating their metacarpophalangeal joint. The subjects were allowed to practice in order to master these movements before the strain measurements were conducted. Each set of measurements comprised five consecutive repetitions of the pressing or plucking movement. A total of five sets were conducted with a 1-min break between sets. Therefore, each subject performed these movements 25 times for each string contact condition.

When the subjects pressed the string forward, the angle formed by three points (the saddle, string contact part, and nut) is defined as $\theta$. The reaction force $F$ on the fingerpad from the string is then given by

$$
F=2 T \cos (\theta / 2) \text {, }
$$

where $T$ is the tension of the string. If a subject pressed the string forward by $10 \mathrm{~mm}$, the calculated reaction force $F$ would be approximately $4.8 \mathrm{~N}$.

\section{Results}

\subsection{Changes in nail strain during string pressing and plucking}

The strain measurement sampling frequency was $1 \mathrm{kHz}$. During the pressing motion for the reference contact condition, the maximum strain occurred around the $1 \mathrm{~s}$ during which the maximum deflection of the string was also generated (Fig. 3). In the axial direction, positive maximum values of strain (tensile strain) were observed at the distal-central and proximal-ulnar parts, whereas negative maximum values (compression strain) were observed at the proximal-radial part (Fig. 3a). In the transverse direction, negative maximum values were found at all locations (Fig. 3b). The string-pressing motion was performed 25 times by each subject, and maximum values at each strain measurement location were calculated from the each string-pressing motion. The mean value of these measurements was used as the maximum strain for each subject.

The results of the changes in the typical nail strain during plucking at the reference contact condition showed that the data obtained for the amount of string movement had stopped partway and could no longer be obtained; however, we assume that the string was separated from the thumb slightly before this event (Fig. 4). Moreover, the $1.3 \mathrm{~mm}$ 


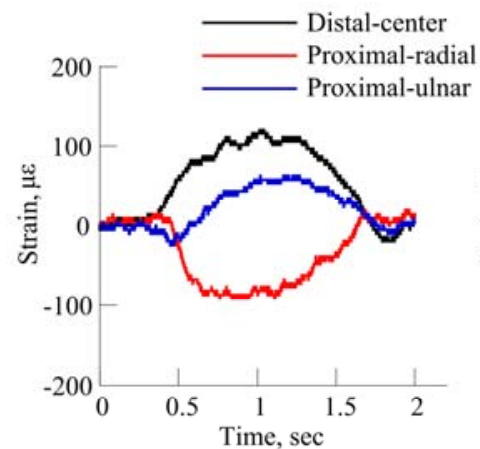

(a) Axial direction

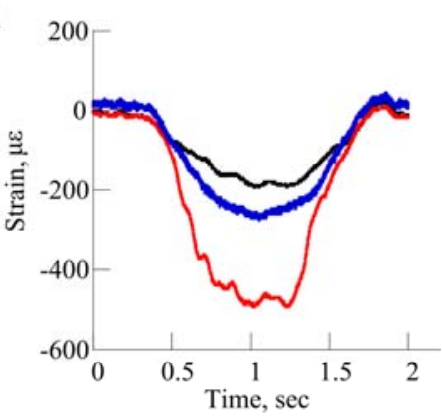

(b) Transverse direction

Fig.3 Typical changes in nail strain during the string-pressing motion. (a) Axial direction, and (b) Transverse direction.

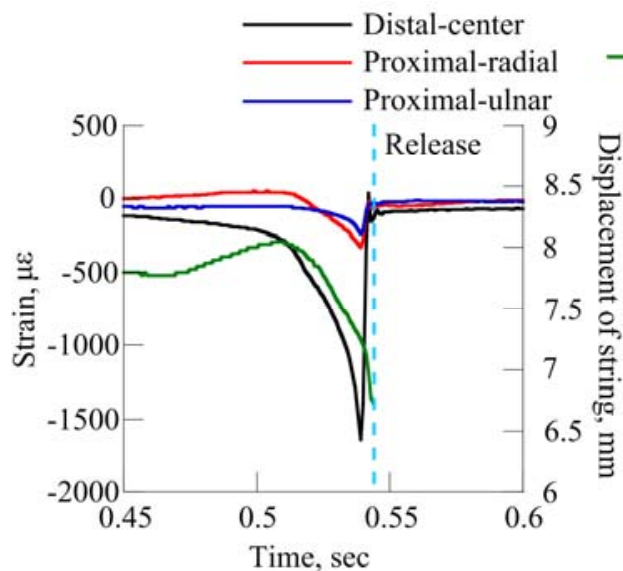

(a) Axial direction

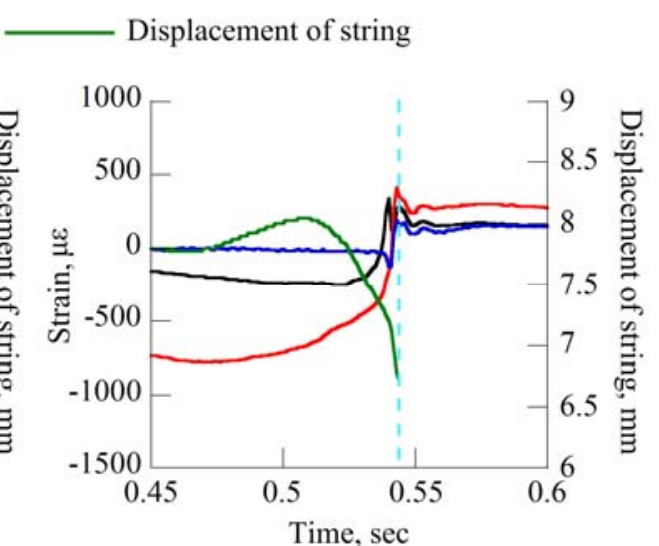

(b) Transverse direction

Fig.4 Typical changes in nail strain during string plucking. (a) Axial direction, and (b) Transverse direction.

decrease in the amount of string movement before the string was separated from the thumb was probably because of the slipping of the string from the thumb surface. When the string slipped from the thumb surface (i.e., decreased amount of string movement), the strain at each measurement location decreased; subsequently, after becoming a negative maximum value, the strain increased (Fig. 4a). The point at which the string stopped affecting the thumb appeared to be when the strain reached its maximum negative value. When the string slipped from the thumb surface, the strain at each measurement location increased and reached the maximum positive value, after which it decreased (Fig. 4b). Similar to the pressing motion, maximum values were calculated from each plucking motion, and the mean value obtained for 25 motions was considered to be the maximum strain for each subject.

\subsection{The effects of measurement location on maximum strain during string pressing and plucking}

The independent variable of measurement location had three levels: radial, central, and ulnar. One-way ANOVA was used for group comparisons, followed by Scheffe's test to determine whether there were significant differences in the maximum strain among the three levels (IBM SPSS Statistics, ver.17.0, Chicago, IL, USA).

The results of our investigation of the effect of measurement location on maximum strain during the string-pressing motion in the reference contact condition indicate that in the axial direction, the maximum strain was significantly lower in the proximal-radial part than those in the distal-central and proximal-ulnar parts $(P<0.01$, Fig. 5a). In fact, asymmetrical strain occurred at both the proximal-radial and proximal-ulnar parts. However, in the transverse direction compression strain occurred at all measurement locations, and no significant differences were observed between locations $(P>0.72)$. The same trend was observed in the other contact conditions. Furthermore, we observed significant asymmetry in the transverse direction at contact angles of $30^{\circ}$ and $60^{\circ}$.

The results of our investigation of the effect of the measurement location on maximum strain during the plucking motion in the reference contact condition indicate that in the axial direction, the maximum strain was significantly 
lower in the distal-central part than in the proximal-radial and proximal-ulnar parts $(P<0.01$, Fig. 5b). No asymmetrical strain was observed in the radial or ulnar directions. However, in the transverse direction, tensile strain was applied at all measurement locations. The maximum strain was significantly higher in the distal-central part than in the proximal-radial and proximal-ulnar parts $(P<0.01)$. The same trend was observed for the proximal and distal contact conditions.

During static motions such as string pressing, we observed asymmetry in thumb nail strain; however, no such asymmetry was observed during dynamic motions such as string plucking.

\subsection{The effects of contact angle on maximum strain during string pressing and plucking}

The independent variable of the contact angle had three levels: $0^{\circ}, 30^{\circ}$, and $60^{\circ}$. We used one-way ANOVA for group comparisons, followed by a Scheffe's test to determine whether or not there were significant differences in the maximum strain among the three levels.

Fig. 6a shows the effect of the contact angle on the maximum strain during the string-pressing motion in the contact condition with the measurement location on the distal-central part and the contact point in the center. The strain significantly $(P<0.01)$ changed from tensile to compression strain in the axial direction, and from compression to tensile strain in the transverse direction with an increase in the contact angle. An almost constant compression strain was applied in the PRT direction irrespective of the contact angle. We observed the same trend for all the other measurement locations.

Fig. $6 \mathrm{~b}$ shows the effect of the contact angle on the maximum strain during the plucking motion in the contact condition with the measurement location on the distal-central part and the contact point in the center. Compression strain significantly $(P<0.01)$ decreased in the axial direction, whereas tensile strain significantly decreased in the transverse direction with an increase in the contact angle. An almost constant strain was applied in the PRA and PUT directions irrespective of the contact angle. We observed the same trend for all the other measurement locations.

\subsection{The effect of contact point on maximum strain during string pressing and plucking}

The independent variable of the contact point had three levels, i.e., proximal, center, and distal. We used one-way ANOVA for group comparisons, followed by a Scheffe's test to determine whether there were significant differences in the maximum strain among the three levels.

Figure $7 \mathrm{a}$ shows the effect of the contact point on the maximum strain during the string-pressing motion for the contact condition with the measurement location on the distal-central part and a contact angle of $0^{\circ}$. The strain significantly $(P<0.01)$ changed from tensile strain to compression strain in the axial direction with a change in the contact point from proximal to distal. However, the strain remained as a compression strain in the transverse direction. The same trend was observed for all the other measurement locations.

Figure $7 \mathrm{~b}$ shows the effect of the contact point on the maximum strain during the plucking motion in the contact condition with the measurement location on the distal-central part and a contact angle of $0^{\circ}$. Compression strain was applied in the axial direction and tensile strain was applied in the transverse direction irrespective of the contact point.

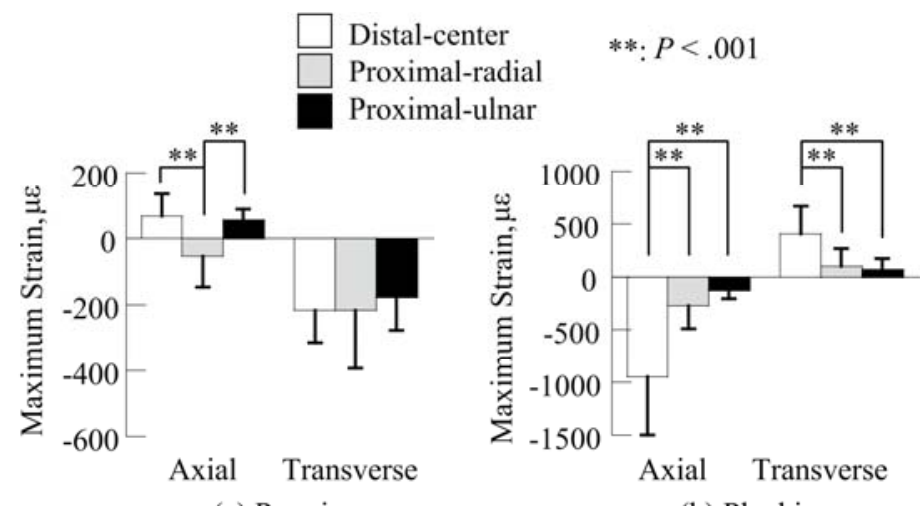

(a) Pressing

(b) Plucking

Fig.5 The effect of measurement location on the maximum strain during string-pressing motion (a) and string plucking motion (b). The contact angle is $0^{\circ}$ and the contact point is in the center. 


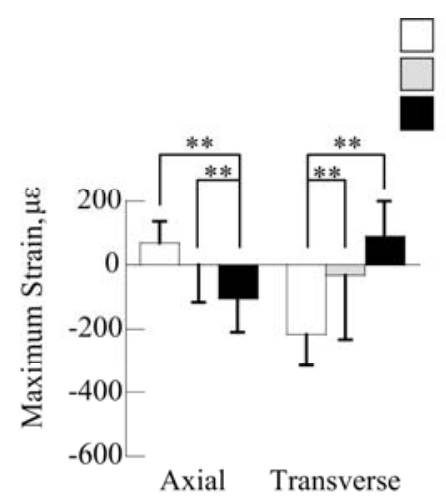

(a) Pressing

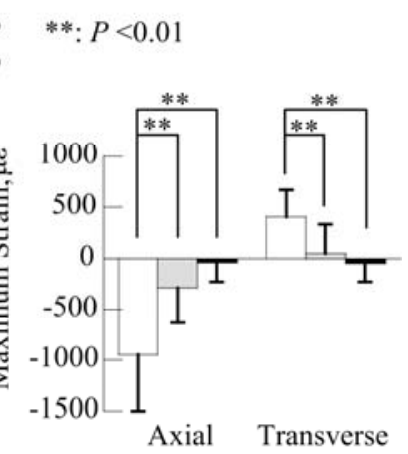

(b) Plucking

Fig.6 The effect of contact angle on the maximum strain during string-pressing motion (a) and string plucking motion (b). The measurement location is the distal-central part and the contact point is in the center.

The same trend was observed for all the other measurement locations.

\section{Discussion}

As shown in Fig. 5a, compression strain was applied in the transverse direction during the string-pressing motion. Meanwhile, tensile strain was applied to the distal-central and proximal-radial parts in the axial direction, and compression strain was applied to the proximal-ulnar part, making the strain applied to the proximal-radial and proximal-ulnar parts asymmetrical. When the thumb pad presses the string, a strong pressure load is applied to the pad at the point of contact with the string, which greatly deforms the thumb pad. This pressure load is transmitted via the thumb pad to the nail. However, although the areas of the thumb pad other than the contact point (areas proximal and distal to the contact point) are deformed, no strong pressure load is applied to the nail. Nail cross-sections in the axial and transverse directions show that the center of the nail bulges and is curved in a crescent shape (Forslind, 1970; Murdan, 2011). As a result, the strong distributed load to correct the curvature is assumed to be applied from the pad to the transverse cross-section of the nail that is in contact with the string. This may be the cause of the compression strain exerted over the nail in the transverse direction. In contrast, in the axial direction, a strong force is applied to the nail in the dorsal direction only at the site of contact with the string. Thus, tensile strain may occur at the distal-central and proximal-radial parts as a result of this part of the nail being pushed in the dorsal direction; however, compression strain occurred at the proximal-ulnar part. A previous study wherein nail strain was measured when the thumb was pressed down onto a flat surface found that strain (compression strain) in the proximal-ulnar-transverse direction is significantly smaller than those in the distal-central-transverse and proximal-radial-transverse directions-an asymmetry identical to that found in the present study (Shimawaki et al., 2006).

There are two likely causes of this asymmetry: (i) asymmetry due to the nail itself and (ii) asymmetry due to the pressure applied to the nail.

The former cause has three likely factors (thickness, degree of curvature, and mechanical properties). No anatomical reports examining the effect of nail thickness and the degree of curvature on asymmetry have yet been published. However, although nail thickness and the degree of curvature cannot be assumed to be perfectly symmetrical, their asymmetry is likely to be very small; therefore, it is extremely unlikely that the strain asymmetry observed in the present study was because nail asymmetry. Furthermore, no studies examining the mechanical properties of the nail in relation to the radioulnar asymmetry have been published. In summary, because not enough research has been conducted related to the nail itself to date, we cannot explain the asymmetry in the strain applied to the nail surface observed in the present study; however, we cannot rule out this possibility and this should therefore be the considered in future studies.

The latter is probably caused by three factors: the thumb pressing force, thumb pad adipose tissue, and the distal phalanx of the thumb). With regard to the thumb pressing force, the side and frontal images taken during this study confirmed that the longitudinal axis of the thumb was almost perpendicular to the string when the thumb is pressed on it. However, taking into consideration the posture of the guitar player while playing the instrument, it was not the precise center of the string halfway between the saddle and the nut $(650 \mathrm{~mm})$ where the thumb pad is pressed down, but a location closer to the saddle (120 $\mathrm{mm}$ from the saddle). As a result, the angle between the longitudinal axis of the 
thumb and the string while it is being pressed downward $10 \mathrm{~mm}$ can be calculated as $85.2^{\circ}$, on the saddle side, and $88.9^{\circ}$ on the nut side; the string does not compress the thumb symmetrically, although this difference is likely to be extremely slight.

The thumb pad adipose tissue is known to be functionally classified into the proximal and distal portions (Shrewsbury and Johnson, 1975, 1983; Gigis and Kuczynski, 1982). The pulp present in the proximal portion is larger and more flexible than that in the distal portion. As a result, the deformation of the proximal pulp when touching an object is greater than that of the distal pulp. This deformation leads to an increase in the contact area with an object, making it possible to securely grasp the object. In contrast, the pulp in the distal portion is rich in fibrous tissue, which limits the amount of deformation and is useful when grasping small objects. In other words, proximal pulp is useful for power gripping, whereas distal pulp is useful for precision gripping (including pinch gripping). In Fig. 7b, we observe that the contact point had no effect on nail strain during string plucking. This is because when the string is in contact with the proximal pulp, the string is held in place by the deformed pulp, which prevents the string from separating from the thumb. As the string gradually moves toward the distal portion, the string separates from the thumb at the point where the distal pulp deformation is weakest. The interaction between the nail, the distal phalanx, and the thumb pulp is well known (Shrewsbury et al., 2003). The distal pulp is firmly attached to the nail plate, and the dorsal surface of the distal phalangeal tuberosity is attached to the nail plate by dense fascia. In this way, the distal pulp, the distal portion of the nail, and the distal phalangeal tuberosity are all firmly attached. By contrast, the proximal portion of the nail rests on top of the nail bed and is not firmly attached. The base and borders on both sides are constrained by the skin. The nail bed is directly associated with the proximal pulp; thus, any displacement constraints are placed upon the nail by the distal pulp and the distal phalangeal tuberosity.

It is known that the shape of the distal phalanx of the thumb is asymmetrical in the radioulnar direction (Shrewsbury et al., 2003). Asymmetric areas include the articular surface of the proximal phalanx, the flexor tendon attachment, and the distal phalangeal tuberosity. On the articular surface of the proximal phalanx, the radial articular surface of the distal phalanx of the thumb is larger in area than the ulnar side and has a deep groove. This allows the distal phalanx of the thumb to pronate toward the index finger during IP joint flexion and allows grasping of objects more securely (Hollister et al., 1995). However, because the IP joint was held still during extension in the present study, it is unlikely to have had a great effect on nail strain asymmetry. The radial ridges of the gable-shaped projection on the flexor tendon attachment of the thumb are longer than the ulnar ridges. This effectively shows that there are many flexor tendon fiber bundles on the radial side; however, this kind of asymmetry is not seen in any of the other fingers and is thought to enhance the force of the distal segment in the direction of rotation. Moreover, this indicates that the structure of the thumb pad is asymmetrical, which may affect nail strain asymmetry. The ulnar spine of the distal phalangeal tuberosity projects more than the radial spine. Given how objects are grasped by the thumb and other fingers, we know that the ulnar side of the thumb pad tends to be the main contact point (Shrewsbury et al., 2003; Kamakura et al., 1980). Thus, the ulnar side of the thumb pad is more frequently used than the radial side, which may cause the asymmetry observed in the spine of the distal phalangeal tuberosity. Because of this, the distal phalanx of the thumb may have become asymmetrical in the radioulnar direction for (a) achieving the opposition mechanism involving the thumb and the other fingers and (b) further enabling a stable and strong power grip or precision grip. Therefore, the underlying cause of the thumbnail strain asymmetry measured in the present study may be the interaction between the pulp, the distal phalanx, and the nail, causing the uniform force applied to the thumb pad to become asymmetrical at the distal phalanx in the radioulnar direction. This asymmetry was then transmitted to the nail.

Changes in the stiffness of the fingerpad pulp that accompany the deformation of the fingerpad pulp may affect the nail strain. Many studies have been conducted on the mechanical deformation of the fingerpad pulp. Peleg et al. (1989) and Serina et al. (1997) addressed the relationship between the deformation of the fingerpad, which occurs when an index finger is pressed against a flat plate and the pressing force. Their results showed that the fingerpad has a nonlinear characteristic of significantly deforming under a low load of $1 \mathrm{~N}$ or less. Shimawaki and Sakai (1997) reported on the relationship between the deformation of the fingerpad and the pressing force, when the angle between the index finger and the flat plate was different, indicating the nonlinear nature of fingerpads. In other words, stiffness is low under a low load, it increases with an increase in the load. This phenomenon likely arises from the structure of the fingerpad pulp. In a previous report (Shimawaki et al., 2013), results from an MRI observation of the deformation of fingerpads have shown that the fat inside the pulp is stored in compartments when there is no load. However, when there is a load, this fat moves to different compartments. When gripping objects or pressing and plucking strings, the 
deformation of the fingerpads toward the back of a hand is limited by the nail. Thus, by deforming as if to wrap around objects and strings, the fingerpads increase the friction with the increased contact area. Further increases in stiffness allows for the stable grip of objects and the pressing/plucking of strings.

According to Fig. 5, the maximum strain in the nail when pressing a string was approximately 150 to $-400 \mu \varepsilon$. Conversely, the maximum strain in the nail when plucking a string was approximately 600 to $1500 \mu \varepsilon$, which represents a notable increase compared to the maximum strain in the nail when pressing a string. When plucking a string, the string movement measured by the laser displacement sensor was approximately $10 \mathrm{~mm}$; therefore, little difference exists in the amount of string movement when pressing or plucking a string; i.e., little difference exists in the reaction force from the string. The mechanism for the difference cannot be speculated from this experiment. However, it is assumed to be due to a rapid recovery of the internal structure of the pad by the string instantly separating from the pad.

As humans developed to become bipedal, they also evolved means of manipulating objects using their hands. During the course of this evolution, asymmetry in the structure of the thumb may have occurred in order to securely grasp objects using the opposable thumb. We measured thumbnail strain when a uniform load was applied to the thumb (pressing on a flat surface and on a string). However, considering power grip and precision grip during which the ulnar surface of the thumb pad is the main point of contact, it is likely that a nonuniform load is applied to the thumb. On the basis of the results of our present and previous thumb nail strain experiments, we assumed that the ulnar side of the thumb pad played an important role in gripping objects. In other words, rather than assuming a uniform structure for the pads and nails, it is necessary to use a more elastic substance than that on the radial surface for the ulnar side of the thumb pad while firmly fixing the ulnar side of the thumb nail to the frame. This allows for a flexible deformation of the ulnar side of the thumb pad (the contact surface with the object). On the other hand, this deformation toward the back of the hand is controlled with the nail. Applying these results to robotic and electric artificial hands should allow even wider contact surfaces. In addition, because the strain of the nail when plucking a string was larger than that when pressing a string, the strain of the nail is assumed to be larger compared to simply grasping an object when robotic hands operate an object. We plan to investigate the relationship between object grasping patterns/operation form and thumbnail strain in a future study.

\section{References}

Baden, H. P., The physical properties of nail, The Journal of Investigative Dermatology, Vol.55, No.2 (1970), pp.115-122.

Ditre, C. M. and Howe, N. R., Surgical anatomy of the nail unit, The Journal of Dermatologic Surgery and Oncology, Vol.18, No.8 (1992), pp.665-671.

Farran, L., Ennos, A. R. and Eichhorn, S. J., The effect of humidity on the fracture properties of human fingernails, The Journal of Experimental Biology, Vol.211, (2008), pp.3677-3681.

Farran, L., Ennos, A. R., Starkie, M. and Eichhorn, S. J., Tensile and shear properties of fingernails as a function of a changing humidity environment, Journal of Biomechanics, Vol.42, No.9 (2009), pp.1230-1235.

Farren, L., Shayler, S. and Ennos, A. R., The fracture properties and mechanical design of human fingernails, The Journal of Experimental Biology, Vol. 207, (2004), pp.735-741.

Fleckman, P. and Allan, C., Surgical anatomy of the nail unit, Dermatologic Surgery, Vol.27, No.3 (2001), pp.257-260.

Forslind, B., Biophysical studies of the normal nail, Acta Dermato-Venereologica, Vol.50, No.3 (1970), pp.161-168.

Gigis, P. L. and Kuczynski, K., The distal interphalangeal joints of human fingers. The Journal of Hand Surgery, Vol.7, No.2 (1982), pp.176-182.

Hollister, A., Giurintano, D. J., Buford, W. L., Myers, L. M. and Novick, A., The axes of rotation of the thumb interphalangeal and metacarpophalangeal joints, Clinical Orthopaedics and Related Research, No.310 (1995), pp.188-193.

Jarrett, A. and Spearman, R. I., The histochemistry of the human nail, Archives of Dermatology, Vol.94, No.5 (1966), pp.652-657.

Jemec, G. E. and Serup, J., Ultrasound structure of the human nail plate, Archives of Dermatology, Vol.125, No.5 (1989), pp.643-646.

Kamakura, N., Matsuo, M., Ishii, H., Mitsuboshi, F. and Miura, Y., Patterns of Static prehension in normal hands, The American Journal of Occupational Therapy, Vol.34, No.7 (1980), pp.437-445. 
Kapandji, I. A., The Physiology of the Joints, Vol. 1 The Upper Limb, 6th ed. (2007), pp.256-257, Churchill Livingstone.

Lewin, K., The normal finger nail, The British Journal of Dermatology, Vol.77, No.8 (1965), pp.421-430.

Mascaro, S. A. and Asada, H. H., Photoplethysmograph fingernail sensors for measuring finger forces without haptic obstruction, IEEE Transactions on Robotics and Automation, Vol.17, (2001), pp.698-708.

Mascaro, S. A. and Asada, H. H., Measurement of finger posture and three-axis fingertip touch force using fingernail sensors. IEEE Transactions on Robotics and Automation, Vol.20, (2004), pp.26-35.

Murdan, S., Transverse fingernail curvature in adults: a quantitative evaluation and the influence of gender, age, and hand size and dominance, International Journal of Cosmetic Science, Vol.33, No.6 (2011), pp.509-513.

Peleg, M. and Campanella, O. H., The mechanical sensitivity of soft compressible testing machines, Journal of Rheology, Vol.33, No.3 (1989), pp.455-467.

Sakai, N. and Shimawaki, S., Motion analysis of thumb in cellular phone use, Applied Bionics and Biomechanics, Vol.7, No.2 (2010), pp.119-122.

Serina, E. R., Mote Jr., C. D. and Rempel, D., Force response of the fingertip pulp to repeated compression - effects of loading rate, loading angle and anthropometry, Journal of Biomechanics, Vol.30, No.10 (1997), pp.1035-1040.

Shimawaki, S. and Sakai, N., Mechanical fingertip deformity responded to the compressive force, Journal of Japanese Society for Clinical Biomechanics, Vol.24 (2003), pp.157-162 (in Japanese).

Shimawaki, S., Sakai, N. and Fukuda, S., Strain measurement of thumbnail in response to compressive force, Transactions of the Japan Society of Mechanical Engineers, Series C, Vol.72, No.718 (2006), pp.1908-1914 (in Japanese).

Shimawaki, S. and Sakai, N., Quasi-static deformation analysis of a human finger using a three-dimensional finite element model constructed from CT images. Journal of Environment and Engineering, Vol.2, No.1 (2007), pp.56-63.

Shimawaki, S., Sakai, N. and Nakabayashi, M., Mechanical deformity of the fingertip and pulp measured using magnetic resonance imaging (MRI), Transactions of the Japan Society of Mechanical Engineers, Series C, Vol.79, No.801 (2013), pp.1709-1717 (in Japanese).

Shrewsbury, M. M. and Johnson, R. K., The fascia of the distal phalanx. The Journal of Bone and Joint Surgery. American Volume, Vol.57, No.6 (1975), pp.784-788.

Shrewsbury, M. M. and Johnson, R. K., Form, function, and evolution of the distal phalanx. The Journal of Hand Surgery, Vol.8, No.4 (1983), pp.475-479.

Shrewsbury, M. M., Marzke, M. W., Linscheid, R. L. and Reece, S. P., Comparative morphology of the pollical distal phalanx, American Journal of Physical Anthropology, Vol.121, No.1 (2003), pp.30-47. 